\title{
Pulmonary Hypertension Associated to Schistosomiasis and Marfan Syndrome
}

Mariana Amélia Gasparetto de Toledo Silva ${ }^{1}$, Fernando Moacyr Fragoso Didier Neto', Karina Moraes Kiso ${ }^{2}$ and Igor Bastos Polonio ${ }^{3 *}$

${ }^{1}$ Resident at Santa Casa School of Medicine, Internal Medicine, São Paulo, Brazil

${ }^{2}$ Assistant Doctor in Internal Medicine Department at Santa Casa School of Medicine, São Paulo, Brazil

${ }^{3}$ Pulmonology Professor and Assistant Doctor at Santa Casa School of Medicine, São Paulo, Brazil

\begin{abstract}
A Case report about a patient with severe pulmonary hypertension which during the investigation non-hepatosplenic schistosomiasis was found, concomitant with pulmonary thromboembolism and Marfan syndrome phenotype is discussed.
\end{abstract}

\section{Introduction}

Pulmonary Hypertension $(\mathrm{PH})$ is a disorder formed by various diseases that have pathological similarities, therefore diverge in pathophysiology and prognosis. Clinically, it is characterized by dyspnea, chest pain and syncope, causing progressive limitation, right-heart failure and death. Histopathologically occurs pulmonary vascular remodeling, with subsequent increase of resistance, and in situ thrombosis [1,2]. Pulmonary Arterial Hypertension (PAH) is defined by the presence of Pulmonary Artery Mean Pressure (mPAP) $\geq 25 \mathrm{mmHg}$, measured by right-heart catheterization, with Pulmonary Artery Occlusion Pressure (PAOP) $\leq 15 \mathrm{mmHg}$ [1-3]. PH is divided in 5 groups, according to the 2008 Dana Point classification: 1 . Pulmonary arterial hypertension, 2. Pulmonary arterial hypertension associated to left-heart diseases, 3. Pulmonary arterial hypertension associated with pulmonary illnesses and/or hypoxia, 4. Pulmonary arterial hypertension associated with chronic thromboembolism, 5. Pulmonary hypertension with uncertain or multifactorial mechanism [4].

Schistosomiasis is one of the major parasitic diseases in the world, endemic in more than 70 countries, especially at those with lower socioeconomic development $(85 \%$ of all the cases in sub-Saharan Africa, India, China and Brazil), estimating 200 million individuals infected worldwide and 10 million in Brazil [1-3]. The lung is a mandatory checkpoint in parasite's lifecycle, which leads to acute and chronic local damage. Hepatoesplenic form, most prevalent form of the chronic illness, characterized by periportal fibrosis and portal hypertension, causes $\mathrm{PH}$ as a well-known complication [2,5]. In fact, the real prevalence of the association between schistosomiasis and $\mathrm{PH}$ is not well defined due to lack of data, with great variability among the publications. Therefore, studies showed up to $7.6 \%$ prevalence of $\mathrm{PH}$ associated to hepatosplenic form, with $4.6 \%$ as $\mathrm{PAH}$ and $3 \%$ as postcapillary $\mathrm{PH}[2,3,5]$. Other studies showed that schistosomal $\mathrm{PH}$ may be the most frequent cause of $\mathrm{PH}$ worldwide, responsible for up to $30 \%$ of all the cases $[1-3,5]$. Schistosomal PH is categorized at group 1- $\mathrm{PAH}$.

Marfan Syndrome (MS) is a connective tissue disease, with autosomal dominant inheritance, related to fibrillin-1 gene (FBN1chromosome 15). It affects especially the musculoskeletal, ocular and cardiovascular systems. Defined by its clinical characteristics, its diagnosis is made according to Ghent's nosology criteria [6,7].

\section{Case Report}

A 27 year-old caucasian male, catholic, single, born in Paraíba-
Brazil, not schooled, unemployed. He complained about progressive fatigue and dyspnea for the last year, with worsening to minimal exertion in the last 6 months, also with nonproductive cough. Reported often lower-limbs edema, especially vespertine, anorexia, and difficult to eat due to dyspnea, with consequent substantial weight loss.

He had medical history of amaurosis secondary to acute glaucoma due to crystalline luxation with 9 years-old, when he had surgery in 1994 (surgical extirpation and prosthetic insertion). He denied any other known comorbidities. He had adequate vaccination, and denied use of any lawful or unlawful drugs, even medicine. Concerning his familiar history, his sister had also amaurosis secondary to acute glaucoma, his mother had systemic arterial hypertension and his grandfather died because of heart disease.

At physical exam: weigh $=51.700 \mathrm{~kg}$, height $=1.60 \mathrm{~m}, \mathrm{BMI}=20.19$ $\mathrm{kg} / \mathrm{m} 2, \mathrm{BP}=110 \times 60 \mathrm{mmHg}, \mathrm{HR}=90 \mathrm{bpm}, \mathrm{BR}=24 \mathrm{bpm}$. Presented in good general condition, hydrated, ruddy, afebrile, anicteric, emaciated, dyspneic, cyanotic lips, jugular distention, although lymphadenopathy was absent. He had pectus excavatum, and thoracic kyphosis. His cardiac rhythm at auscultation was regular, therefore with hyperphonesis and split of S2 in pulmonic area; the respiratory auscultation was normal; liver was palpable $5 \mathrm{~cm}$ from the right costal margin; his extremities had palpable and symmetric pulses, feet edema and slight clubbing and cyanosis, arachnodactyly and disproportionately long limbs too.

The patient was admitted for investigation and clinical compensation. PH (Pulmonary Hypertension) was diagnosed after right-heart catheterization that revealed reduced cardiac index and output, elevation of systemic and pulmonary vascular resistance and the following results: mean pulmonary arterial pressure $=60$ $\mathrm{mmHg}$; Pulmonary artery occlusion pressure $=28 \mathrm{mmHg}$; Right atrial pressure $=16 \mathrm{mmHg}$.

Various tests were performed, based on algorithms proposed by $\mathrm{PH}$

${ }^{*}$ Corresponding author: Dr. Igor Bastos Polonio, Professor of Pulmonology, Santa Casa School of Medicine, São Paulo, Brazil, E-mail: igbpolonio@gmail.com

Received March 04, 2013; Accepted March 27, 2013; Published March 29, 2013

Citation: de Toledo Silva MAG, Didier Neto FMF, Kiso KM, Polonio IB (2013) Pulmonary Hypertension Associated to Schistosomiasis and Marfan Syndrome. J Hypertens 2: 112. doi:10.4172/2167-1095.1000112

Copyright: () 2013 de Toledo Silva MAG, et al. This is an open-access article distributed under the terms of the Creative Commons Attribution License, which permits unrestricted use, distribution, and reproduction in any medium, provided the original author and source are credited. 
guidelines. The first echocardiography indicated significant dilation of the right chambers, small pericardial effusion, paradoxal movement of the interventricular septum, mild to severe tricuspid insufficiency, with estimated Pulmonary Artery Systolic Pressure (PASP) of $90 \mathrm{mmHg}$. Kato-Katz technique on feces revealed 24 eggs per field. Abdominal ultrasonography showed homogeneous hepatomegaly related to an enlarged right lobe and hepatic veins ectasia. Thoracic computed tomography indicated small right pleural effusion, cardiomegaly mainly related to the right chambers, mild pericardial effusion (Figure 1), pulmonary artery trunk in the superior limit of normality (Figure 2), areas compatible with mosaic perfusion, and multiple lack of contrast filling in the interlobar arteries and its branches, with many collateral circulation, and also peripheral lingula consolidation, compatible with pulmonary infarction.

The patient was treated with warfarin, furosemide, dygoxin, supplementary $\mathrm{O} 2$, enalapril and bosentan. After optimizing therapeutics and with greater compensation of the symptoms, the patient was discharged, but still needing supplementary $\mathrm{O} 2$ at home and waiting for pulmonary transplantation. The complaints worsened and got refractory to the treatment. The patient had hemoptysis, hemothorax, cardiogenic shock and then died at the hospital.

\section{Discussion}

The patient had clinical condition compatible with $\mathrm{PH}$, which was confirmed by right-heart catheterization. The procedure revealed disproportional postcapilar reactive $\mathrm{PH}$ (transpulmonary index $>12$ ), in other words, there were pre and post-capilar components causing hypertension [8].

Furthermore, test for schistosomiasis was positive. However,

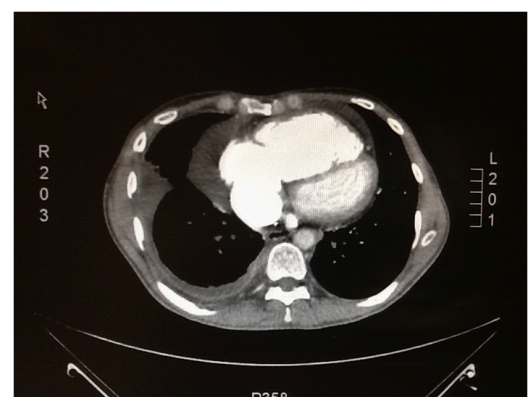

Figure 1: Cardiomegaly with right ventricle and atrium dilation, pericardial and pleural effusion.

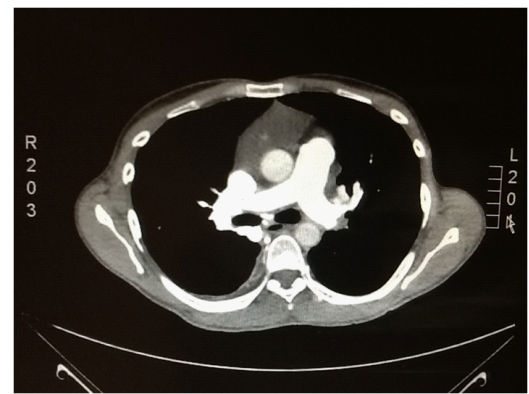

Figure 2: Pulmonary artery trunk in the superior limit of normality. hepatoesplenic form, commonly related to $\mathrm{PH}$, wasn't confirmed by abdominal ultrasonography. It has been demonstated that the presence of hepatic changes is not necessary for occurrence of pulmonary involvement, since it follows multiple mechanisms, still in research: mechanical impaction (embolism) of the parasite eggs in pulmonary arteries; inflammatory process and vascular remodeling; high pulmonary blood output due to arteriovenous shunts in presence of portal hypertension $[2,5]$.

There are difficulties on diagnosing Marfan Syndrome (MS) due to findings also present in general healthy population; some of those, however, are uncommon but frequent in MS, like crystalline luxation, present in $50-80 \%$ of the patients [6]. Our patient's background of ocular illness, added to other musculoskeletal characteristics aroused suspicion of MS. It has been asked then if MS was contributing for $\mathrm{PH}$ in that patient. It is known that some collagenosis can cause PH. MS is not described as a possible cause of $\mathrm{PH}$, but instead of its rarity, it has been described the association of these two diseases. It is related, for example, a case that a patient was treated as a bearer of idiopathic $\mathrm{PH}$ for years, and later discovered that he had MS, with pulmonary artery dilation [9].

Likewise it was evidenced the presence of pulmonary thrombosis which when associated to schistosomiasis occurs because of the great dilation of pulmonary vessels, slowing of blood flow and consequent formation of thrombosis in situ at arterial inner wall. In this case, however, pulmonary artery trunk was on superior limit of normality and CT scan evidenced thrombo embolism. We believe that the probable origin of this embolus is the right ventricle, since it was very enlarged and liable to thrombi formation. The presence of pulmonary thrombosis was also a determinant of the clinical worsening.

Other characteristic concerning the prognosis is the presence of pericardial effusion in this case, already established as independent severity factor on $\mathrm{PH}[3]$.

\section{References}

1. Lapa MS, Ferreira EV, Jardim C, Martins Bdo C, Arakaki JS, et al. (2006) Clinical characteristics of pulmonary hypertension patients in two reference centers in the city of Sao Paulo. Rev Assoc Med Bras 52: 139-143.

2. Correa Rde A, Moreira MV, Saraiva JM, Mancuzo EV, Silva LC, et al. (2011) Treatment of schistosomiasis-associated pulmonary hypertension. J Bras Pneumol 37: 272-276.

3. Souza R, Cardoso AP, Pedra CA, Jardim C, Watge D, et al. (2005) Diretrizes Brasileiras para Manejo da Hipertensão Pulmonar. J Bras Pneumol 31: S1. S31.

4. Simonneau G, Robbins IM, Beghetti M, Channick RN, Delcroix M, et al. (2009) Updated clinical classification of pulmonary hypertension. J Am Coll Cardio 54: S43-54.

5. Hovnanian A, Hoette S, Fernandes CJ, Jardim C, Souza R (2010) Schistosomiasis associated pulmonary hypertension. Int J Clin Pract Suppl: 25-28.

6. Yuan SM, Jing H (2010) Marfan's syndrome: an overview. Sao Paulo Med $J$ 128: 360-366.

7. De Paepe A, Devereux RB, Dietz HC, Hennekam RC, Pyeritz RE (1996) Revised diagnostic criteria for the Marfan syndrome. Am J Med Genet 62: 417 426.

8. Hoette S, Jardim C, Souza Rd (2010) Diagnosis and treatment of pulmonary hypertension: an update. J Bras Pneumol 36: 795-811.

9. Ting $\mathrm{P}$, Jugdutt $\mathrm{BI}$, Le Tan $\mathrm{J}$ (2010) Large pulmonary artery aneurysm associated with Marfan syndrome. Int J Angiol 19: e48-50. 